\title{
Tanshinone IIA Ameliorates Streptozotocin-Induced Diabetic Nephropathy, Partly by Attenuating PERK Pathway-Induced Fibrosis
}

This article was published in the following Dove Press journal: Drug Design, Development and Therapy

\author{
Shujuan $\mathrm{Xu}^{1}, *$ \\ Lianjun $\mathrm{He}^{2, *}$ \\ Keke Ding ${ }^{3}$ \\ Lingling Zhang ${ }^{4}$ \\ Xinhui $\mathrm{Xu}^{5}$ \\ Sheng Wang ${ }^{6}$ \\ Xueyi Qian ${ }^{2}$ \\ 'Department of Surgery, Yijishan \\ Hospital of Wannan Medical College, \\ Wuhu, People's Republic of China; \\ ${ }^{2}$ Precision Medicine Centre, Yijishan \\ Hospital of Wannan Medical College, \\ Wuhu, People's Republic of China; \\ ${ }^{3}$ Department of Urology, Yijishan \\ Hospital of Wannan Medical College, \\ Wuhu, People's Republic of China; \\ ${ }^{4}$ School of Pharmacy, Southwest Medical \\ University, Luzhou, People's Republic of \\ China; ${ }^{5}$ School of Pharmacy, Wannan \\ Medical College, Wuhu, People's Republic \\ of China; ${ }^{6}$ Department of Pharmacy, \\ Yijishan Hospital of Wannan Medical \\ College, Wuhu, People's Republic of \\ China
}

*These authors contributed equally to this work
Purpose: Tanshinone IIA (Tan IIA), a compound extracted from Salvia miltiorrhiza, can improve type II diabetes, while the molecular mechanisms underlying Tan IIA-mediated protective effects in diabetic nephropathy are unclear. This study explored the protective actions of Tan IIA on renal tissues in streptozotocin (STZ)-induced diabetic nephropathy.

Materials and Methods: Tan IIA (2, 4, $8 \mathrm{mg} / \mathrm{kg} / \mathrm{day})$ was daily administered to STZtreated rats by intraperitoneal injection for 42 days. The morphologic pathology was evaluated by hematoxylin-eosin and Masson's trichrome staining, and transmission electron microscopy. The protein expression levels in renal tissues were evaluated by Western blotting and immunohistochemistry; the mRNA expression level was determined by quantitative realtime PCR.

Results: Tan IIA at 2 and $4 \mathrm{mg} / \mathrm{kg}$ attenuated the increase in the levels of uric acid and blood urea nitrogen and restored the reduction in the superoxide dismutase activity in the serum of the diabetic rats. Tan IIA at 2 and $4 \mathrm{mg} / \mathrm{kg}$, but not $8 \mathrm{mg} / \mathrm{kg}$, ameliorated the thickening of renal tubule in the diabetic rats; Tan IIA at 2 and 4 and $8 \mathrm{mg} / \mathrm{kg}$ attenuated the thickening of glomerular basement membrane and the collagen deposition in the renal tissues of the diabetic rats. Tan IIA treatment at $2,4,8 \mathrm{mg} / \mathrm{kg}$ decreased the expression levels of transforming growth factor-beta1, TSP-1, Grp78 and CHOP in the diabetic rats. Tan IIA at 2 and 4 and $8 \mathrm{mg} / \mathrm{kg}$ attenuated the increase in the protein levels of p-PERK, p-elf $2 \alpha$ and ATF-4 from the renal tissues of diabetic rats, while the protein level of AFT- 6 and the mRNA expression levels of XBP-1t, XBP-1s and p58IPK in the renal tissues were not affected by STZ or Tan IIA treatment.

Conclusion: Tan IIA-mediated protective effects on the STZ-induced diabetic nephropathy may be associated with the reduced endoplasmic reticulum stress via attenuating PERK signaling activities.

Keywords: diabetic nephropathy, streptozotocin, tanshinone IIA, renal tissues, endoplasmic reticulum stress, PERK signaling

\section{Introduction}

Diabetes mellitus (DM) is a main health threat with severe complications. Diabetic nephropathy (DN) represents one of the most common diabetic complications with its incidence being increased annually worldwide. It has been reported that DN affects up to $40 \%$ of all diabetic patients. ${ }^{1} \mathrm{DN}$ is characterized by glomerular hypertrophy and increased renal tubular thickness, which ultimately leads to renal failure. Indeed, tubulointerstitial fibrosis that displays an excessive extracellular 
matrix $(E C M)$ in renal tissue has been observed in most advanced DN patients. ${ }^{2}$ However, multiple factors and pathways are contributed to excessive accumulation of ECM components including inflammatory factors, growth factors, angiogenic factors and proteinase. ${ }^{3}$ Among these, transforming growth factor-beta1 (TGF- $\beta 1$ ) is regarded to be the major mediator of ECM expression. ${ }^{4,5}$ TGF- $\beta 1$ is synthesized by tubular and epithelial cells, which can promote the ECM production through distinct intracellular signals. ${ }^{4,5}$ Although the full mechanism of the expression of TGF- $\beta 1$ under hyperglycemia condition remains uncertain, an increasing body of literature implies that endoplasmic reticulum (ER) stress acts as a key player in promoting the activation of TGF- $\beta 1 .{ }^{6}$

The ER is an organelle and regulates the protein folding process. Under normal physiological conditions, the demand for protein folding and the capacity of unfolded proteins degradation are well balanced. However, ER homeostasis is disrupted under hyperglycemic stimulation, which results in the accumulation of unfolded or misfolded proteins. $^{7}$ Initially, unfolded protein response (UPR) is triggered to minimize ER-stress activation-induced proteotoxicity; while glucose-regulated protein 78 (Grp78) involves in activating the UPR sensors. Under ER stress, the binding of Grp78 to misfolded or unfolded proteins is increased and in the meantime, Grp78 dissociates from protein kinase RNA (PKR)-like ER kinase (PERK), inositol requiring $1 \alpha$ (IRE1 $\alpha$ ), and activating transcription factor 6 (ATF-6), then activates growth arrest and DNA damageinducible gene 153 (CHOP), which is a key marker for ER stress. ${ }^{8}$ Although these three sensors participate in different signaling pathways, there is cross-talk between the different pathways, and all of them are associated with TGF- $\beta 1$ expression, ${ }^{9-11}$ however, how these signaling pathways regulate the activation of TGF- $\beta 1$ activation under the pathology of DN remains unknown.

Tanshinone IIA (Tan IIA) is mainly derived from Salvia miltiorrhiza. Tan IIA exerts multiple effects such as anti-tumor and anti-angiogenic activities. ${ }^{12,13}$ Studies found that Tan IIA could diminish the collagen deposition in human cardiac fibroblast and inhibit renal tubular epithelial cell fibrosis induced by high glucose. ${ }^{14}$ Mechanistically, the anti-fibrosis effects of Tan IIA were associated with suppression of TGF- $\beta$ pathway signaling activities. Furthermore, Tan IIA attenuated TGF$\beta 1$-mediated fibrosis through suppressing the ER stress in cardiac fibroblasts. ${ }^{15}$ However, up to date, the molecular mechanisms underlying Tan IIA-mediated protective effects in diabetic nephropathy are unclear. Therefore, this study is mainly aimed to determine the protective actions of Tan IIA on renal tissues in streptozotocin (STZ)-induced diabetic nephropathy and to explore the underlying molecular mechanisms.

\section{Materials and Methods \\ Reagents}

Streptozotocin (STZ) was obtained from Alexis Corporation (ALX-380-010-G001; Alexis Corporation, Lausen, Switzerland). Glucose Electrode Calibrator (Model: ACCU-CHEK) was acquired from Roche Products Limited (Basel, Switzerland). Tan IIA was purchased from Aladdin Corporation (\#337,919-71-8; Shanghai, China). Primary antibodies including Grp78 (\#3177), TGF- $\beta 1$ (\#3709), thrombospondin-1 (TSP-1; \#37,879), p-PERK (\#3179), ATF-4 (\#11,815), ATF-6 $(\# 65,880)$, phosphorylated eukaryotic initiation factor 2 alpha (p-elf2 $\alpha ; \# 3398)$ and $\beta$-actin (\#\#4970) were purchased from Cell Signaling Technology (Danvers, USA). CHOP antibody (\#ab10444) was purchased from Abcam (Cambridge, USA).

\section{Animals}

The animal experimental procedures were in accordance with the National Institutes of Health Guide and approved by the Committee for the Care and Use of Yijishan Hospital of Wannan Medical College. Fifty male SpragueDawley (SD) rats (165-185 g) were obtained from the Zhejiang Chinese Medical University (Hangzhou, China). Animals were housed under standard conditions: $22^{\circ} \mathrm{C}$, a 12-h light/12-h dark cycle, standard food and water ad libitum.

\section{Establishment of STZ-Induced Diabetic Model and Tan IIA Treatment}

Type 1 diabetes animal model was induced as previously described. ${ }^{16}$ Rats were fasted $12 \mathrm{~h}$, and the animals were intraperitoneally injected with STZ $(10 \mathrm{mM}$ citrate buffer with $\mathrm{pH}=4.5$ ) at a dosage of $60 \mathrm{mg} / \mathrm{kg}$ for consecutive 2 days. At 2-day post-STZ injection, rat tail vein blood glucose levels were detected by using a Glucose Electrode Calibrator, and diabetic rats were defined as a blood glucose level greater than $16.7 \mathrm{mM}$. For the control group, the rats were injected intraperitoneally with $10 \mathrm{mM}$ citrate buffer with $\mathrm{pH}=4.5$ at a volume of $2 \mathrm{~mL} / \mathrm{kg}$. The rats were randomly assigned into five 
groups: control group, diabetes group, and diabetes with three dosages of Tan IIA injection group $(2,4,8 \mathrm{mg} / \mathrm{kg} /$ day, $\mathrm{n}=10$ for each group), and each group had 10 animals. Dimethyl sulfoxide (DMSO) was used to dissolve Tan IIA, and the DMSO-prepared Tan IIA was diluted to $10 \%$ by $0.9 \%$ saline before use. For the Tan IIA treatments, rats were intraperitoneally injected with respective doses of Tan IIA daily for 42 days. Rats from the control and diabetes groups were treated with $0.9 \%$ saline $(2 \mathrm{~mL} /$ $\mathrm{kg}$, intraperitoneal injection) daily for 42 days.

\section{Biochemical Measurements}

Rats were anesthetized intraperitoneal injection of $30 \mathrm{mg} /$ $\mathrm{kg}$ pentobarbital sodium salt (\#P3761; Sigma-Aldrich) after 42-day treatments. Blood was taken from carotid arteries and the serum from the blood samples was collected by centrifugation at $1500 \mathrm{~g}$ for $15 \mathrm{~min}$ at $4^{\circ} \mathrm{C}$. Serum samples were kept at $-20^{\circ} \mathrm{C}$ for evaluation of serum creatinine ( $\mathrm{sCr}$ ), uric acid (UA), blood urea nitrogen (BUN) and superoxide dismutase (SOD) activity. The levels of sCr, BUN and UA were measured by an automatic biochemical analyser (Siemens AG; Munich, Germany). The SOD was determined by the commercial kit (\#ab65354; Abcam) according to the manufacturer's protocol.

\section{Hematoxylin-Eosin and Masson's Trichrome Staining}

The left kidneys from the rats were removed, and after 24 $\mathrm{h}$ fixation in $4 \%$ paraformaldehyde (PFA), the tissues were embedded in paraffin. The tissues were then cut into $5 \mu \mathrm{m}$ sections followed by staining with hematoxylin-eosin (H\&E). Collagen in the kidney tissues was evaluated by Masson's trichrome staining according to previous methods. ${ }^{17}$ The stained sections were viewed and recorded using a light microscope $(400 \times$ amplification; Nikon, Japan). Thickness of the renal tubule and collagen deposition was quantified using the Image J program (NIH) by two pathologists, who are blinded to the treatments.

\section{Immunohistochemistry}

Sections (5 $\mu \mathrm{m}$-thick) of kidney tissues were deparaffinized, rehydrated and immersed in $10 \mathrm{mM}$ citric acid (pH 6.0) for antigen retrieval. The sections were incubated with primary antibodies against Grp78 (1:100), CHOP (1:50), TGF- $\beta 1$ (1:100) and TSP-1 (1:100) overnight at $4^{\circ} \mathrm{C}$, followed by incubating with biotinylated goat anti- rabbit IgG (1:200, \# BA1003; Boster Biological Technology, Wuhan, China) in phosphate-buffered saline (PBS) for $2 \mathrm{~h}$ at room temperature. After a further incubation in avidin-biotin-peroxidase complex solution (ABC, 1:100, Boster Biological Technology, Wuhan, China) for $2 \mathrm{~h}$ at room temperature, the respective antigens were visualized with $0.05 \%$ 3,3'-diaminobenzidine (DAB; \#D5905; Sigma-Aldrich) with H\&E counterstaining. Each section was scanned using a Panoramic MIDI (3DHISTECH, Hungary). IHC staining was scored independently by two pathologists [percentage of positive cells: five categories: $0(<10 \%), 1(10-25 \%), 2(25-50 \%)$, $3(50-75 \%)$, and $4(75-100 \%)$; intensity: four categories (from low to high): $0,1,2$, and 3; and a final IHC staining score (intensity score $\times$ percentage score)] was determined. ${ }^{18}$

\section{Transmission Electron Microscopy}

Sections from the renal cortex area of the right kidneys were diced into 1-2 $\mathrm{mm}$ pieces and fixed in 4\% PFA for 4 $\mathrm{h}$ followed by post-fixation with $1 \%$ osmium tetroxide for $30 \mathrm{~min}$. Then, sections were then dehydrated in graded alcohol, transferred to propylene oxide and embedded in Epon 812 resin in blocks. Eighty nanometer sections were collected using a diamond histoknife (Diatome) on an Ultracut E microtome (Leica), and then mounted on a copper mesh and stained with uranyl acetate and lead nitrate. The UTHSCSA Image Tools 3.0 (University of Texas Medical School at San Antonio) was used to determine the thickness of the glomerular basement membrane. Glomerular basement membrane (GBM) thickness was determined by the orthogonal intercept method. ${ }^{19}$

\section{Quantitative Real-Time Polymerase Chain Reaction (qRT-PCR)}

Total RNA was isolated from the renal tissues of the rats after different treatments using a TRIzol reagent kit (\#12,094,346; Invitrogen, Carlsbad, USA) following the protocol of the manufacturer. The concentration and quality of the RNA were assessed by absorbance spectrometry measuring absorbance ratios of A260/ A280 and A260/A230 using a NanoDrop ND-1000 spectrophotometer (Thermo Fisher Scientific, Waltham, USA). A total of $1 \mu \mathrm{g}$ RNA was reverse transcribed into complementary DNA (cDNA) using a PrimeScript 1st strand cDNA Synthesis Kit (\#6110A; TaKaRa, Japan). The real-time PCR was performed using the $\mathrm{SYBR}^{\circledR}$ 
Table I Sequences of Primers for qRT-PCR

\begin{tabular}{|c|c|c|}
\hline Gene & Forward (5'-3') & Reverse (5'-3') \\
\hline Grp-78 & GCATCCTGGTGGCTTTCCAGCCATTC & CTGGGTACATTTGATCTGACTGG \\
\hline $\mathrm{CHOP}$ & СTGCTCСTTCTCСTTCATGC & AGCAGAGGTCACAAGCACCT \\
\hline$X B P-I s$ & AAACAGAGTAGCAGCGCAGACTGC & GGATCTCTAAAACTAGAGGCTTGGTG \\
\hline$X B P-I t$ & GAGTCCGCAGCAGGTG & GTGTCAGAGTCCATGGGA \\
\hline$p 58^{I P K}$ & TCCTGGTGGACCTGCAGTACG & CTGCGAGTAATTTCTTCCCC \\
\hline GAPDH & GGTGGACCTCATGGCCTACAT & GCСTCTCTCTTGCTCTCAGTATCCT \\
\hline
\end{tabular}

Premix Ex Taq ${ }^{\mathrm{TM}}$ (\#RR820B; Takara) on an ABI7900 Biosystems (Applied Biosystems, Foster City, USA). Oligonucleotide primer sequence is showed in Table 1. PCR efficiency and melting peak integrity was checked for each run. Each sample was assayed in triplicates. The relative mRNA expression levels were calculated using the comparative $\mathrm{Ct}$ method using the GAPDH as the internal reference.

\section{Western Blot Analysis}

Renal tissues were homogenized with ice-cold radioimmunoprecipitation assay buffer according to the manufacturer's instructions. Total protein concentration was measured by a BCA Protein Assay Reagent Kit (\#23,225; Pierce Biotechnology, Rockford, USA). A total of $30 \mu \mathrm{g}$ protein from each sample was resolved on a $10 \%$ SDS-PAGE gel and was transferred to a PVDF membrane (\#IPVH00010; Millipore Corporation, Billerica, USA) at $18 \mathrm{~V}$ for $1 \mathrm{~h}$ via the semi-dry transfer method. The membranes were blocked with $5 \%$ non-fat dry milk at $37^{\circ} \mathrm{C}$ for $1 \mathrm{~h}$, and the proteins were probed against respective primary antibodies accordingly to previously published methods. ${ }^{18}$ The $\beta$-actin was used as the internal control.

\section{Statistical Analysis}

All results expressed as median (min-max). The results were analyzed by Kruskal-Wallis test followed by Dunn's multiple comparisons test. A P value less than 0.05 was considered statistically significant.

\section{Results \\ Plasma Glucose Level Was Reduced by Tan IIA Treatment}

No significant difference was detected in plasma glucose concentration and body weight in these five groups before Tan IIA treatment (Table 2). At 6 weeks after STZ-treatment, the body weight of the rats was significantly reduced when compared to the control rats (Table 2); while Tan IIA treatment at 2 and $4 \mathrm{mg} / \mathrm{kg}$ significantly increased the body weight in the diabetic rats when compared to the untreated diabetic ones (Table 2); however, Tan IIA treatment at $8 \mathrm{mg}$ / $\mathrm{kg}$ failed to prevent the body weight loss in the diabetic rats (Table 2). In addition, the level of plasma glucose was higher in the diabetes group than that in the control group (Table 2); while 2 and $4 \mathrm{mg} / \mathrm{kg}$ Tan IIA but not $8 \mathrm{mg} / \mathrm{kg}$ Tan IIA significantly reduced the plasma glucose level in the diabetic rats when compared to the untreated diabetic group (Table 2).

Table 2 The Effect of Tanshinone IIA (Tan IIA) on Body Weight and Blood Glucose Concentration

\begin{tabular}{|c|c|c|c|c|}
\hline \multirow[t]{2}{*}{ Groups } & \multicolumn{2}{|l|}{ Body Weight (g) } & \multicolumn{2}{|c|}{ Blood Glucose Concentration (mmol/L) } \\
\hline & $\begin{array}{l}\text { Before Tan IIA } \\
\text { Injection }\end{array}$ & $\begin{array}{l}\text { After } 6 \text { Weeks Tan IIA } \\
\text { Injection }\end{array}$ & $\begin{array}{l}\text { Before Tan IIA } \\
\text { Injection }\end{array}$ & $\begin{array}{l}\text { After } 6 \text { Weeks Tan IIA } \\
\text { Injection }\end{array}$ \\
\hline Control & $132.65(\mid 27.8-135.7)$ & $410.1(365.4-457.3)$ & $5.3(5.1-6.4)$ & $5.5(5.0-6.4)$ \\
\hline Diabetes model & $134.2(127.4-140.6)$ & $335.4(270.4-358.9)^{*}$ & $25.3(25.0-26.7)^{*}$ & $25.5(20.9-28.7)^{*}$ \\
\hline $\begin{array}{l}\text { Tan IIA (2 mg/kg) } \\
(2 \mathrm{mg} / \mathrm{Kg})\end{array}$ & $136.4(|29.3-| 40.7)$ & $329.6(294.1-382.0)^{*}, \#$ & $26.2(25.22-7.3)$ & $19.5(17.0-23.8)^{*}, \#$ \\
\hline $\begin{array}{l}\text { Tan IIA }(4 \mathrm{mg} / \mathrm{kg}) \\
(4 \mathrm{mg} / \mathrm{Kg})\end{array}$ & $131.55(\mid 22.2-140.3)$ & 331.91 (287.6-391.2)*,\# & $26.0(25.12-6.5)$ & $22.1(17.0-25.6)^{*}, \#$ \\
\hline $\begin{array}{l}\text { Tan IIA (8 mg/kg) } \\
(8 \mathrm{mg} / \mathrm{Kg})\end{array}$ & $131.2(|22.7-14| .7)$ & $317.7(240.7-381.6)^{*}$ & $26.3(25.1-27.2)$ & $23.6(17.0-29.4)^{*}$ \\
\hline
\end{tabular}

Notes: Data were presented as median (min - max; $n=10) .{ }^{*} P<0.05$ vs control group; ${ }^{\#} P<0.05$ vs diabetes group. 


\section{Tan IIA Treatment Improves the Renal Function of the Diabetic Rats}

As shown in Figure 1A, no significant difference was detected in $\mathrm{sCr}$ levels among these five groups (Figure 1A). Both UA and BUN levels were increased in the diabetes group comparing to the control group (Figure $1 \mathrm{~B}$ and $\mathrm{C}$ ). Tan IIA treatment at 2,4 and $8 \mathrm{mg} / \mathrm{kg}$ significantly reduced UA levels when compared to the diabetes group (Figure 1B); 2 and $4 \mathrm{mg} / \mathrm{kg}$ Tan IIA but not $8 \mathrm{mg} / \mathrm{kg}$ Tan IIA significantly reduced the BUN level when compared to the diabetes group (Figure 1C). In addition, the SOD level in the diabetes group was decreased when compared to the control group (Figure 1D); 2 and $4 \mathrm{mg} / \mathrm{kg}$ Tan IIA but not $8 \mathrm{mg} / \mathrm{kg}$ Tan IIA significantly increased the SOD level when compared to the diabetes group (Figure 1D).
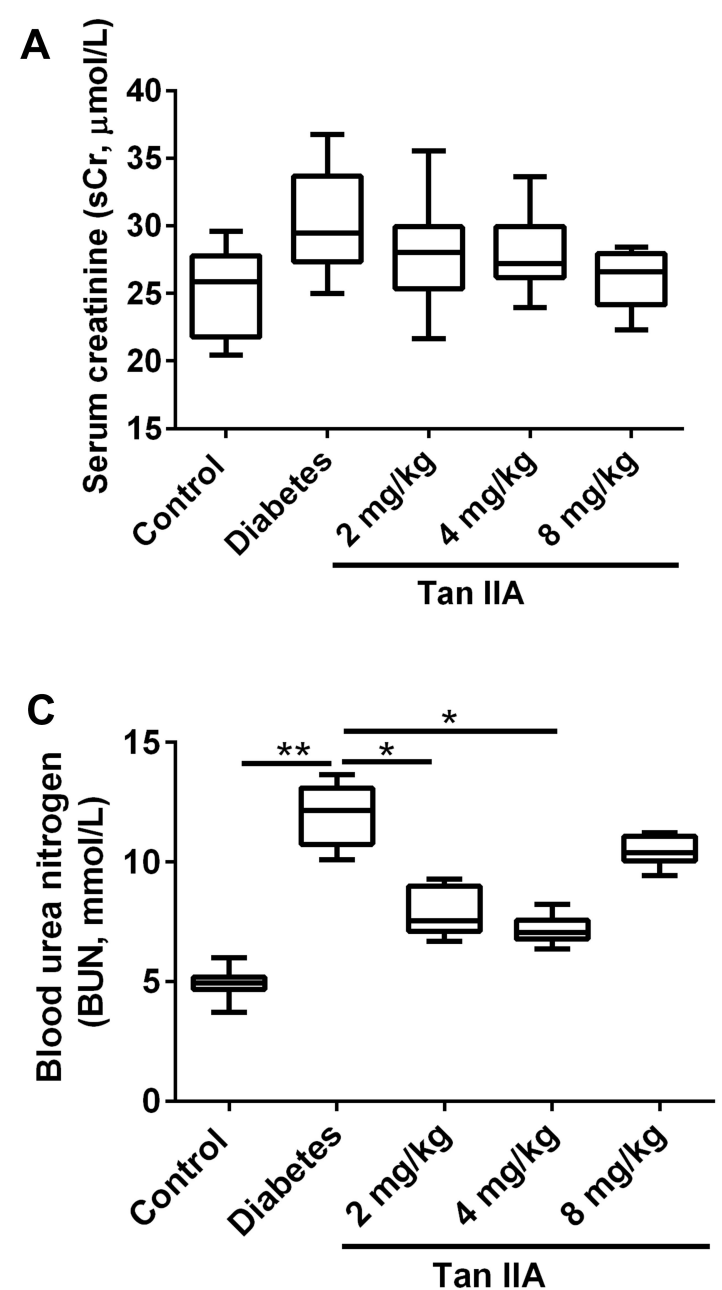

\section{Tan IIA Ameliorates the Pathological Changes in the Renal Tissues of the Diabetic Rats}

As shown in Figure 2A, the thickness of the renal tubules in the diabetes group was significantly increased when compared to the control group (Figure 2A); while 2 and $4 \mathrm{mg} / \mathrm{kg}$ Tan IIA but not $8 \mathrm{mg} / \mathrm{kg}$ Tan IIA significantly decreased the thickness of the renal tubules including the proximal and distal duct when compared to the diabetes group (Figure 2A). Furthermore, by transmission electron microscopy (TEM), thickening of the glomerular basement membrane (GBM) was clearly increased in the diabetes group when compared to the control group (Figure 2B); Tan IIA treatment at 2, 4 and $8 \mathrm{mg} / \mathrm{kg}$ significantly reduced the thickness of GBM when compared to the diabetes group. In addition,
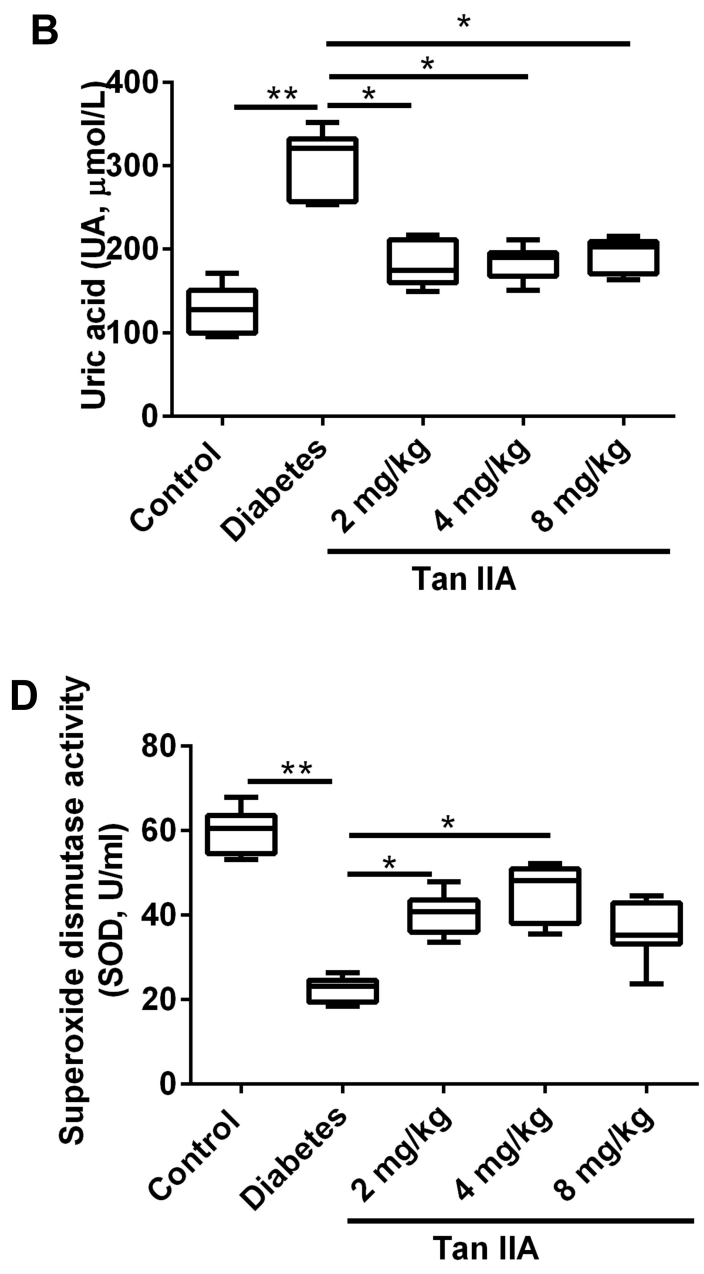

Figure I Tan IIA treatment improves the renal function of the diabetic rats. (A) The levels of serum creatinine (sCr), (B) Uric acid (UA) and (C) Blood urea nitrogen (BUN) in different treatment groups were determined. (D) The level of SOD activity in different treatment groups was determined by SOD activity assay kit. $\mathrm{N}=10$; $*$ P $<0.05$ and $* * \mathrm{P}<0.01$. 


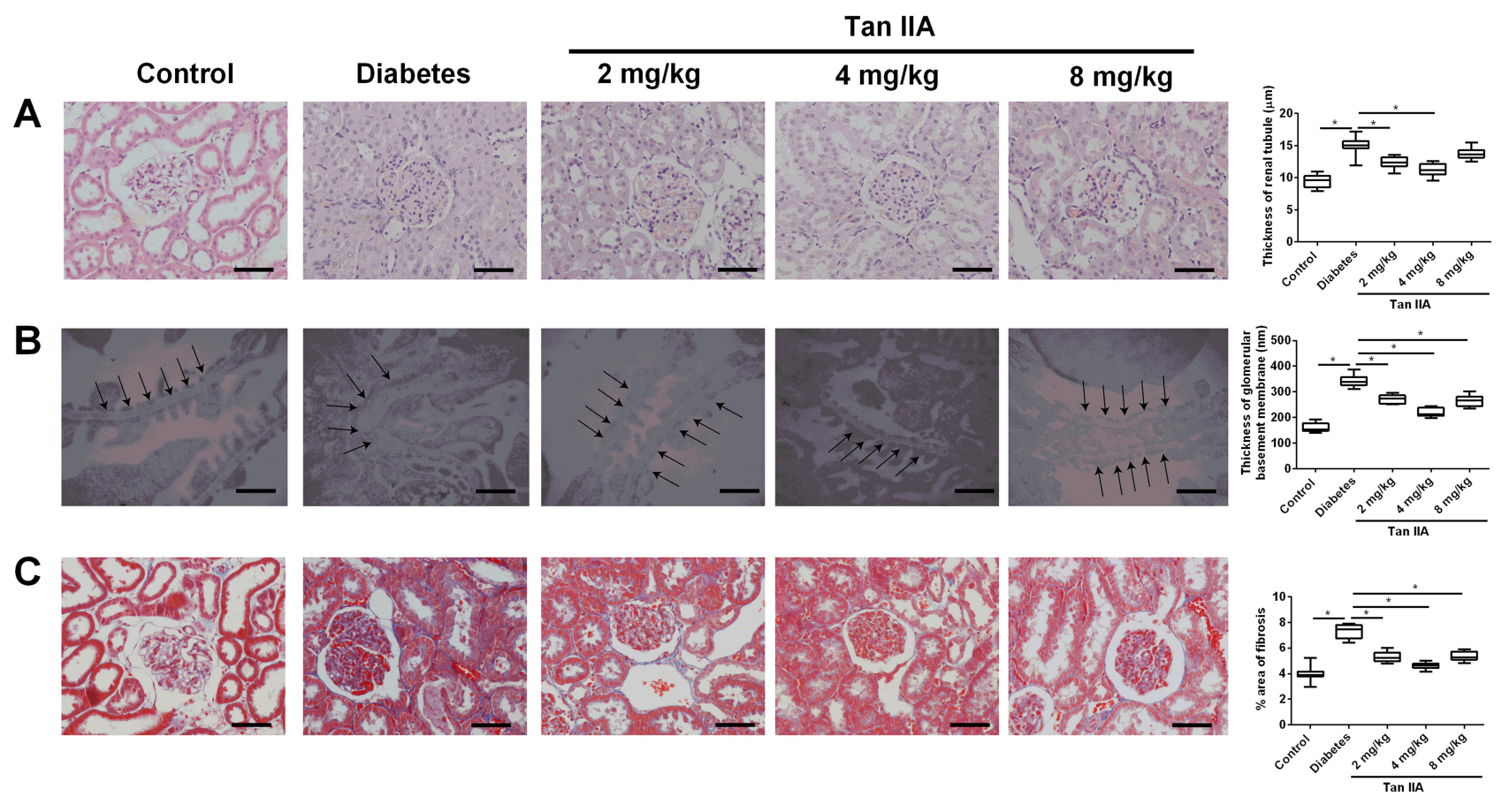

Figure 2 Tan IIA ameliorates the pathological changes in the renal tissues of the diabetic rats. (A) Thickness of renal tubules among different treatment groups were determined by HE staining. Scale bar: $50 \mu \mathrm{m}(\times 400)$. (B) Thickening of glomerular basement membrane (GBM, indicated by arrows) among different treatment groups was determined by TEM. The pooled data from five sections for each group is summarized. Scale bar: $2 \mu \mathrm{m}(\times 7900)$. (C) The distribution of collagens (blue color) stained with Masson's trichrome staining in renal tissue from different groups was evaluated. Scale bar: $50 \mu \mathrm{m}(\times 400) . \mathrm{N}=10 ; * \mathrm{P}<0.05$.

Masson' staining revealed that collagen (blue staining) was widely expressed in the tubular lumen and glomeruli of diabetic rats (Figure 2C), and the percentage of collagen area was significantly higher in the diabetic group than that in the control group (Figure 2C). Tan IIA treatment $(2,4,8 \mathrm{mg} / \mathrm{kg})$ reduced the collagen formation in the renal tissues of the diabetic rats when compared to the diabetes group (Figure 2C).

\section{Tan IIA Down-Regulates TGF- $\beta$ I, TSP-I, Grp78 and CHOP Expression in the Renal Tissues of the Diabetic Rats}

The IHC results showed that the protein expression levels of TGF- $\beta 1$, TSP-1, Grp78 and CHOP were significantly higher in the diabetes group compared with the control group (Figure 3A). Tan IIA treatment at $2,4,8 \mathrm{mg} / \mathrm{kg}$ all significantly decreased the protein levels of TGF- $\beta 1$, TSP1, Grp78 and CHOP in the diabetic rats (Figure 3A). Consistently, the qRT-PCR results showed that the mRNA expression of Grp78 and CHOP was significantly up-regulated in the diabetes group when compared to the control group (Figure 3B and C); while Tan IIA treatment at $2,4,8 \mathrm{mg} / \mathrm{kg}$ significantly attenuated the increase in the
Grp78 and CHOP mRNA expression level in the renal tissues from the diabetic rats (Figure $3 \mathrm{~B}$ and $\mathrm{C}$ ).

\section{Tan IIA Attenuates the Activation of ER Stress via PERK Pathway in the Diabetic Rats}

There are three main pathways that could be involved in ER stress activation. To dissect the exact mechanism leading to attenuate fibrosis by Tan IIA, the protein or gene expression levels of specific molecules in these different pathways were examined. The key proteins (p-PERK, p-elf2 $\alpha$ and ATF4) involved in the PERK pathways were determined in different groups (Figure 4A). The Western blot results showed that the protein expression levels of p-PERK, p-elf $2 \alpha$ and ATF-4 in diabetic rats were significantly increased when compared to the control group (Figure 4A and C); while Tan IIA treatment at 2, 4, $8 \mathrm{mg} / \mathrm{kg}$ all significantly decreased the protein levels of p-PERK, p-elf $2 \alpha$ and ATF-4 (Figure 4A). In contrast, there is no significant difference among the five groups in the protein expression level of activated ATF6 (Figure 4B). In addition, for the IRE1 pathway, there is no significant difference among the five groups in the mRNA expression levels of both XBP-1t, XBP-1s and p58 ${ }^{\mathrm{IPK}}$ (Figure 4C). 


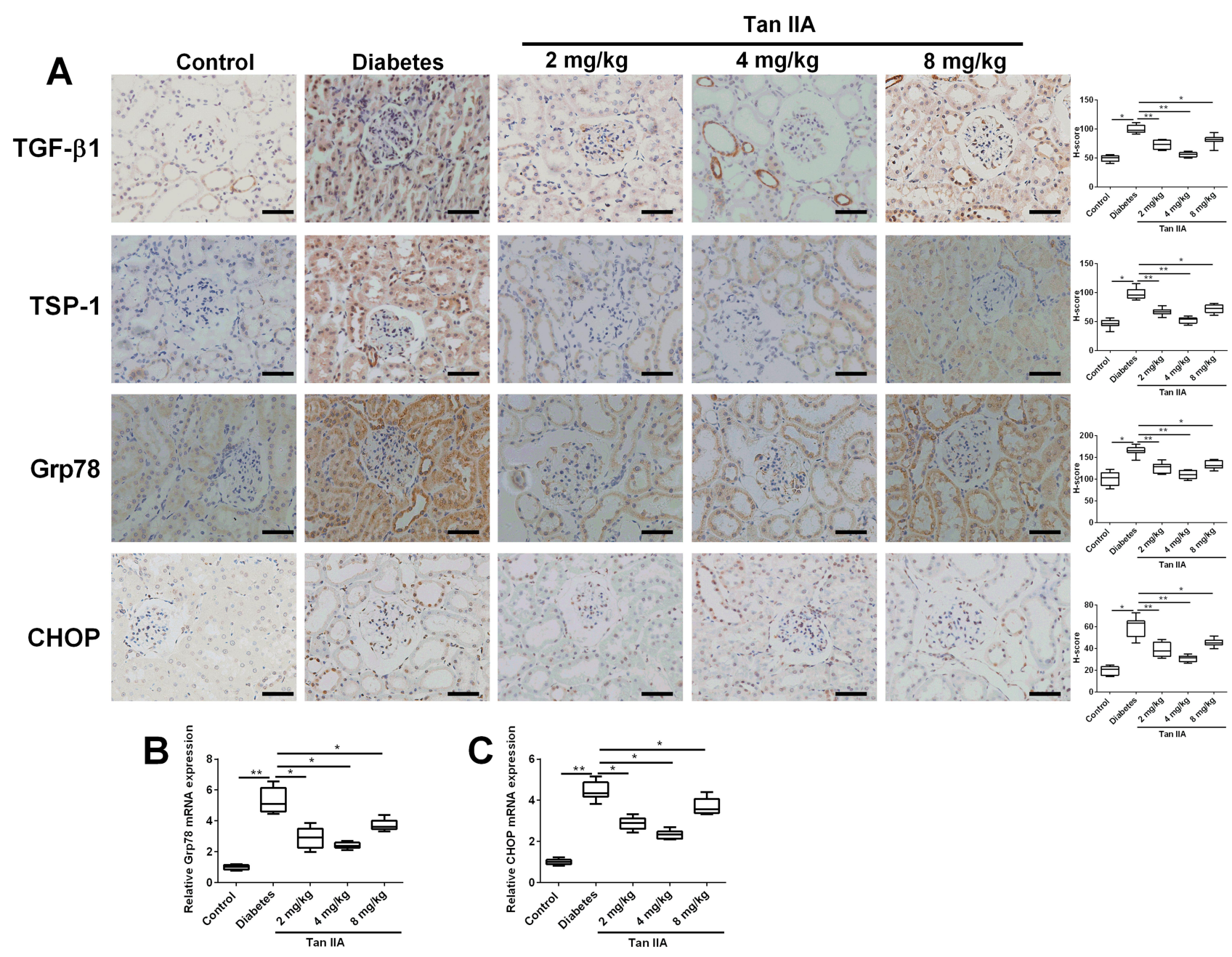

Figure 3 Tan IIA down-regulates TGF- $\beta$ I, TSP-I, Grp78 and CHOP expression in the renal tissues of the diabetic rats. (A) Immunohistochemical analysis of TGF- $\beta$ I, TSP-I, Grp78 and CHOP expression in the renal tissues among five groups and the pooled data from ten sections for each group is summarized. Scale bar: $50 \mu \mathrm{m}(\times 400)$. (B, C) The expression levels of Grp78 (B) and CHOP $(\mathbf{C})$ from different treatment groups were determined by qRT-PCR. N = I0; $* P<0.05$ and $* * P<0.01$.

\section{Discussion}

This study evaluated the protective actions of Tan IIA on renal function in diabetic rats. The results showed that body weight and plasma glucose concentration were reduced in Tan IIA treatment groups with low dosages $(2,4 \mathrm{mg} / \mathrm{kg})$, it suggested that Tan IIA was involved in glucose metabolism which was consistent with other studies. ${ }^{20,21}$ However, plasma glucose level did also not alter by $8 \mathrm{mg} / \mathrm{kg} /$ day Tan IIA treatment, which might be due to cytotoxic effects of high dose of Tan IIA. ${ }^{22}$

It is well known that $\mathrm{DN}$ is characterized by increased thickening of the renal tubule which was initially pathological change of fibrosis. ${ }^{23}$ In the present study, increased thickening of the renal tubules was observed in STZinduced diabetes by HE staining. ${ }^{24}$ It is reported that podocytes have been found to contribute to glomerular hypertrophy. Under normal conditions, podocytes have a very limited capacity to proliferate; however, podocytes undergo hypertrophy by increasing cell size with longterm uncontrolled hyperglycemic situation. ${ }^{25}$ The results showed that Tan IIA with low dosages treatment could reduce the renal tubules thickening and attenuate glomerular hypertrophy.

According to previous studies, these histopathological changes, including glomerular hypertrophy and narrowing renal tubular lumen, were associated with increased ECM expression and GBM thickening. ${ }^{26,27}$ Therefore, GBM thickening was detected by TEM. The result showed that administration of Tan IIA had a beneficial effect on ameliorating GBM thickening; however, the underlying mechanism has not been investigated. Collagen is one of the major constituents of ECM in GBM and localizes within the GBM matrix network. Therefore, we hypothesized that Tan IIA may be associated with the suppression 
A
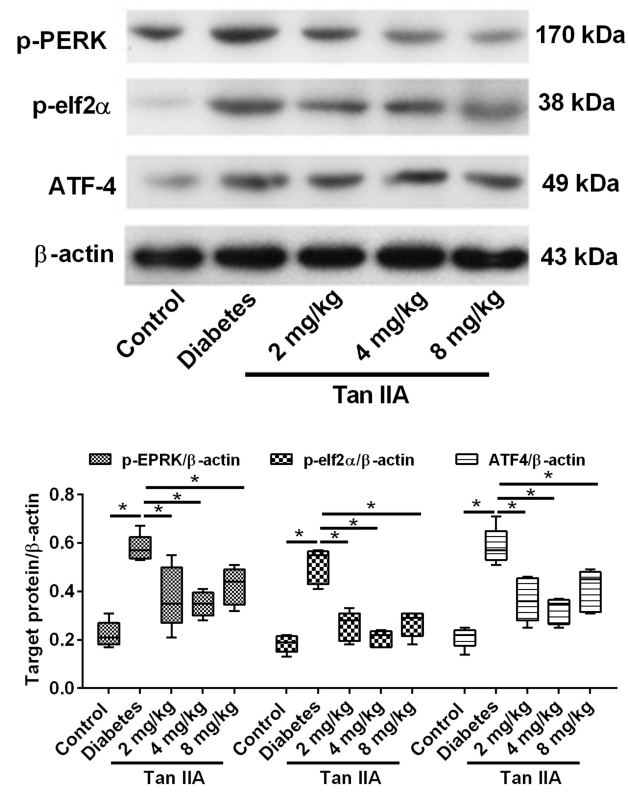

C

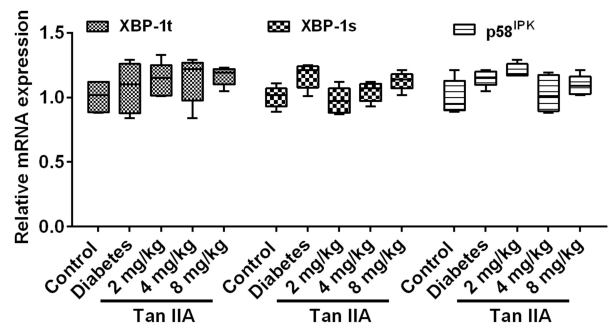

B
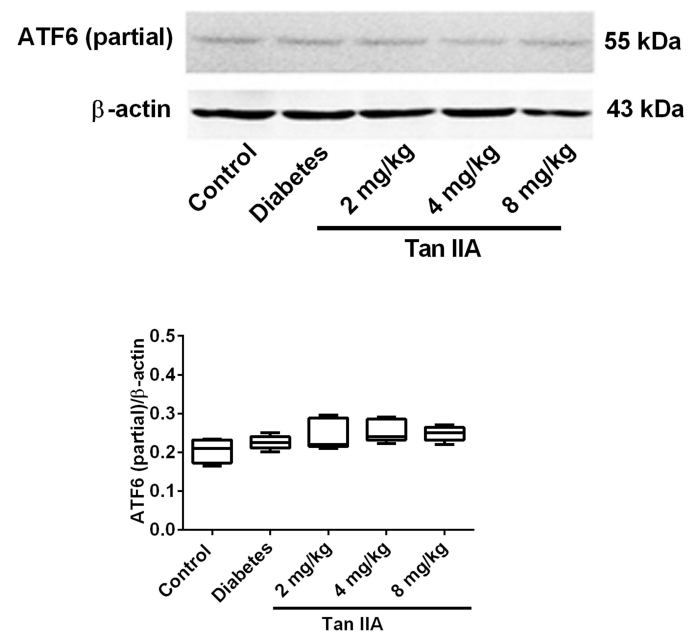

Figure 4 Tan IIA reduces renal fibrosis through the perk pathway rather than the IREI and ATF6 pathways. (A) p-perk, p-eif2a and ATF4 protein expression levels were detected by Western blot in renal tissue in different treatment groups. (B) The expression of active ATF6 was detected by Western blot in renal tissue in different treatment groups. (C) The mRNA expression levels of XBP-It, XBP-Is and p58IPK were detected by qRT-PCR. $N=5 ; * P<0.05$.

of collagen expression. Masson's staining revealed that hyperglycemia-induced an increase in the collagen in the tubular lumen and glomeruli of diabetic rats, which was attenuated by Tan IIA treatment.

According to a previous study, over-expression of TGF- $\beta 1$ contributed to collagen deposition and GBM thickening. ${ }^{28}$ Additionally, TGF- $\beta 1$ can mediate tubulointerstitial fibrosis and renal hypertrophy in $\mathrm{DN}^{29}$ Our results showed that the diabetic state enhanced renal TGF- $\beta 1$ expression, which is consistent with other reports. $^{30}$ TGF- $\beta 1$ can bind to TGF- $\beta$ receptor II (T $\beta$ RII), which can activate multiple pathways, and TSP-1 has been found to involve in these pathways. The down-regulated TSP-1 gene resulted in less fibrosis of renal tissues. ${ }^{31}$ In our study, Tan IIA suppressed the expression of TGF- $\beta 1$ and TSP-1, suggesting that the anti-fibrosis effect of Tan IIA might be associated with the TGF- $\beta 1 /$ TSP- 1 pathway. Although the protective effects of Tan IIA on suppressing the progress of fibrosis via TGF- $\beta 1$ expression have been confirmed in previous literatures, ${ }^{17,20}$ the full mechanism is still unclear.

Previous studies suggest that ER stress activation acts as a key player in renal fibrosis development. ${ }^{32}$ Once the homeostasis is dysregulated under cellular stress conditions such as oxidative stress, the accumulation of unfolded and misfolded proteins in the ER could cause ER stress. In our results, SOD activity was decreased in diabetic rats, it suggested that oxidative stress may be triggered. After ER stress activation, Grp78 is dissociated from PERK, IRE1 and ATF-6 to bind with the unfolded or misfolded proteins. A recent study showed that ER stress inhibitors protected against renal fibrosis by alleviating Grp78 expression. ${ }^{33}$ In the present study, we observed that Tan IIA suppressed the TGF- $\beta 1$ and TSP-1 expression levels via decreasing the expression of Grp78 and CHOP. As discussed above, PERK pathway, IRE1 pathway and ATF-6 pathway are all involved in the expression of TGF$\beta 1 ;^{9-11}$ however, there is few work to determine the 
underlying mechanism of increased TGF- $\beta 1$ induced by ER stress.

Our results also showed that the expression levels of p-PERK, p-elf2 and ATF-4 were all enhanced in renal tissue of diabetic rats. PERK phosphorylates eukaryotic initiation factor 2 alpha (elf $2 \alpha$ ) and leads to regulate ATF4 , which encodes TGF- $\beta 1$ transcription. However, the expressions of XBP-1s, p58IPK and active ATF-6 did not alter, which suggested that the IRE1 $\alpha-\mathrm{XBP} 1$ and ATF-6 pathways are probably not involved. Therefore, the increased expression of TGF- $\beta 1$ may be due to the activation of the PERK pathway, but not the IRE1 and ATF6 pathways in our model. One potential explanation is that, according to some previous studies, the IRE $1 \alpha-\mathrm{XBP} 1$ and ATF-6 pathways are inactivated in cells undergoing prolonged ER stress, whereas PERK signaling is sustained. ${ }^{34-36}$ Therefore, in the renal tissue of diabetes, increased expression of TGF- $\beta 1$ was mainly reduced by Tan IIA treatment via suppressing PERK pathway.

Finally, some parameters of renal function including BUN and UA were evaluated. BUN is one of the main terminal products of protein metabolism and UA is a product of purine metabolism. However, both are excreted via glomerular filtration. Increased concentration of BUN and UV indicates the decreased renal function and the possibility of renal failure. ${ }^{37}$ The concentration of serum UA and BUN levels in serum were decreased after Tan IIA application; these results implied that Tan IIA was able to rescue kidney function in DN.

The present study still has its own limitations. The present study lacks the results showing that lower glucose level can rescue the pathology of DN, and future studies may consider to treat the diabetic rats with insulin to further confirm the effects of glucose levels on the pathophysiology of DN. The present study has not measured urinary protein excretion, which is also a key marker for renal dysfunction, and future studies should measure this parameter. Moreover, the protective effects of Tan IIA were mainly observed at 2 and $4 \mathrm{mg} /$ $\mathrm{kg}$, but not at $8 \mathrm{mg} / \mathrm{kg}$, and this may be explained by that Tan IIA at $8 \mathrm{mg} / \mathrm{kg}$ may exceed the physiological dosage, and this dosage may exert non-specific effects or even toxic effects on the renal tissues of the diabetic rats. Further studies are required to fully understand the differential effects of Tan IIA at different doses on the rental functions in DN.

\section{Conclusions}

In conclusion, the present study showed that Tan IIA attenuated STZ-induced renal dysfunction and morphologic pathology in DN rats. The protective effects of Tan IIA may be associated with the suppression of PERK pathway activation and collagen expression via elevation of SOD activity. This study further provides promising role of Tan IIA in DN treatment, and may guide the use of Tan IIA in clinical patients.

\section{Statement of Ethics}

The animal experimental procedures were in accordance with the National Institutes of Health Guide and approved by the Committee for the Care and Use of Yijishan Hospital of Wannan Medical College.

\section{Acknowledgments}

This research was supported by University Natural Science Research Project of Anhui Province (Grant No. KJ2018A0252).

\section{Disclosure}

The authors report no conflicts of interest for this work.

\section{References}

1. Lam TH, Ho DS, Jiang C, NCD Risk Factor Collaboration. Worldwide trends in diabetes since 1980: a pooled analysis of 751 population-based studies with 4.4 million participants. Lancet (London, England). 2016;387(10027):1513-1530. doi:10.1016/S014 0-6736(16)00618-8

2. Kolset SO, Reinholt FP, Jenssen T. Diabetic nephropathy and extracellular matrix. J Histochem Cytochem. 2012;60(12):976-986. doi: $10.1369 / 0022155412465073$

3. Bonnans C, Chou J, Werb Z. Remodelling the extracellular matrix in development and disease. Nat Rev Mol Cell Biol. 2014;15 (12):786-801. doi:10.1038/nrm3904

4. Sutariya B, Jhonsa D, Saraf MN. TGF- $\beta$ : the connecting link between nephropathy and fibrosis. Immunopharmacol Immunotoxicol. 2016;38 (1):39-49. doi:10.3109/08923973.2015.1127382

5. Tang F, Hao Y, Zhang X, Qin J. Effect of echinacoside on kidney fibrosis by inhibition of TGF- $\beta 1 /$ Smads signaling pathway in the $\mathrm{db} / \mathrm{db}$ mice model of diabetic nephropathy. Drug Des Devel Ther. 2017;11:2813-2826. doi:10.2147/DDDT.S143805

6. Xu Y, Wang J, Pan T, et al. Role of the ER stress in prostaglandin E2/ E-prostanoid 2 receptor involved TGF- $\beta 1$-induced mice mesangial cell injury. Mol Cell Biochem. 2016;411(1-2):43-55. doi:10.1007/s11010015-2567-z

7. Fan Y, Lee K, Wang N, He JC. The role of endoplasmic reticulum stress in diabetic nephropathy. Curr Diab Rep. 2017;17(3):17. doi:10.1007/s11892-017-0842-y

8. Chen Y, Gui D, Chen J, He D, Luo Y, Wang N. Down-regulation of PERK-ATF4-CHOP pathway by astragaloside IV is associated with the inhibition of endoplasmic reticulum stress-induced podocyte apoptosis in diabetic rats. Cell Physiol Biochem. 2014;33(6):1975-1987. doi:10.1159/000362974

9. Heindryckx F, Binet F, Ponticos M, et al. Endoplasmic reticulum stress enhances fibrosis through IRE1 $\alpha$-mediated degradation of miR-150 and XBP-1 splicing. EMBO Mol Med. 2016;8(7):729-744. doi:10.15 252/emmm.201505925 
10. Koo JH, Lee HJ, Kim W, Kim SG. Endoplasmic reticulum stress in hepatic stellate cells promotes liver fibrosis via PERK-mediated degradation of HNRNPA1 and up-regulation of SMAD2. Gastroenterology. 2016;150(1):181-193.e188. doi:10.1053/j.gastro.2015.09.039

11. Hoffman SM, Tully JE, Nolin JD, et al. Endoplasmic reticulum stress mediates house dust mite-induced airway epithelial apoptosis and fibrosis. Respir Res. 2013;14(1):141. doi:10.1186/1465-9921-14-141

12. Li Q, Hu K, Tang S, Xu LF, Luo YC. Anti-tumor activity of tanshinone IIA in combined with cyclophosphamide against lewis mice with lung cancer. Asian Pac J Trop Med. 2016;9(11):1084-1088. doi:10.1016/j.apjtm.2016.09.003

13. Tsai MY, Yang RC, Wu HT, Pang JH, Huang ST. Anti-angiogenic effect of tanshinone IIA involves inhibition of matrix invasion and modification of MMP-2/TIMP-2 secretion in vascular endothelial cells. Cancer Lett. 2011;310(2):198-206. doi:10.1016/j.canlet.2011.06.031

14. Cao L, Huang B, Fu X, Yang J, Lin Y, Lin F. Effects of tanshinone IIA on the regulation of renal proximal tubular fibrosis. Mol Med Rep. 2017;15(6):4247-4252. doi:10.3892/mmr.2017.6498

15. Gu J, Li H-L, Wu H-Y, et al. Sodium tanshinone IIA sulfonate attenuates radiation-induced fibrosis damage in cardiac fibroblasts. $J$ Asian Nat Prod Res. 2014;16(9):941-952. doi:10.1080/10286020.2014.935769

16. Xu L, Shen P, Bi Y, et al. Danshen injection ameliorates STZ-induced diabetic nephropathy in association with suppression of oxidative stress, pro-inflammatory factors and fibrosis. Int Immunopharmacol. 2016;38:385-394. doi:10.1016/j.intimp.2016.06.024

17. Jiang C, Shao Q, Jin B, Gong R, Zhang M, Xu B. Tanshinone IIA attenuates renal fibrosis after acute kidney injury in a mouse model through inhibition of fibrocytes recruitment. Biomed Res Int. 2015;2015:867140. doi:10.1155/2015/867140

18. Zhang Q, He L, Dong Y, et al. Sitagliptin ameliorates renal tubular injury in diabetic kidney disease via STAT3-dependent mitochondrial homeostasis through SDF-1 $\alpha /$ CXCR4 pathway. FASEB J. 2020;34 (6):7500-7519. doi:10.1096/fj.201903038R

19. Zhang H, Nair V, Saha J, et al. Podocyte-specific JAK2 overexpression worsens diabetic kidney disease in mice. Kidney Int. 2017;92 (4):909-921. doi:10.1016/j.kint.2017.03.027

20. Kim SK, Jung KH, Lee BC. Protective effect of tanshinone IIA on the early stage of experimental diabetic nephropathy. Biol Pharm Bull. 2009;32(2):220-224. doi:10.1248/bpb.32.220

21. Chen J, Bi Y, Chen L, Zhang Q, Xu L. Tanshinone IIA exerts neuroprotective effects on hippocampus-dependent cognitive impairments in diabetic rats by attenuating ER stress-induced apoptosis. Biomed Pharmacother. 2018;104:530-536. doi:10.1016/j.biopha.2018.05.040

22. Chiu TL, Su CC. Tanshinone IIA induces apoptosis in human lung cancer A549 cells through the induction of reactive oxygen species and decreasing the mitochondrial membrane potential. Int J Mol Med. 2010;25(2):231-236.

23. Najafian B, Alpers CE, Fogo AB. Pathology of human diabetic nephropathy. Contrib Nephrol. 2011;170:36-47.
24. Bose M, Almas S, Prabhakar S. Wnt signaling and podocyte dysfunction in diabetic nephropathy. J Clin Invest Med. 2017;65 (8):1093-1101. doi:10.1136/jim-2017-000456

25. Petermann AT, Pippin J, Durvasula R, et al. Mechanical stretch induces podocyte hypertrophy in vitro. Kidney Int. 2005;67 (1):157-166. doi:10.1111/j.1523-1755.2005.00066.x

26. Erensoy N, Yilmazer S, Oztürk M, Tunçdemir M, Uysal O, Hatemi H. Effects of ACE inhibition on the expression of type IV collagen and laminin in renal glomeruli in experimental diabetes. Acta Histochem. 2004;106(4):279-287. doi:10.1016/j.acthis.2004.04.001

27. Barton M, Sorokin A. Endothelin and the glomerulus in chronic kidney disease. Semin Nephrol. 2015;35(2):156-167. doi:10.1016/j. semnephrol.2015.02.005

28. Kumar GS, Salimath PV. Effect of spent turmeric on kidney glycoconjugates in streptozotocin-induced diabetic rats. J Diabetes Metab Disord. 2014;13:78. doi:10.1186/2251-6581-13-78

29. Castro NE, Kato M, Park JT, Natarajan R. Transforming growth factor $\beta 1$ (TGF- $\beta 1$ ) enhances expression of profibrotic genes through a novel signaling cascade and microRNAs in renal mesangial cells. J Biol Chem. 2014;289(42):29001-29013. doi:10.1074/jbc.M114.600783

30. Lo CS, Shi Y, Chang SY, et al. Overexpression of heterogeneous nuclear ribonucleoprotein F stimulates renal Ace-2 gene expression and prevents TGF- $\beta 1$-induced kidney injury in a mouse model of diabetes. Diabetologia. 2015;58(10):2443-2454. doi:10.1007/s00125-015-3700-y

31. Bige N, Shweke N, Benhassine S, et al. Thrombospondin-1 plays a profibrotic and pro-inflammatory role during ureteric obstruction. Kidney Int. 2012;81(12):1226-1238. doi:10.1038/ki.2012.21

32. Kim H, Moon SY, Kim JS, et al. Activation of AMP-activated protein kinase inhibits ER stress and renal fibrosis. Am J Physiol Renal Physiol. 2015;308(3):F226-F236. doi:10.1152/ajprenal.00495.2014

33. Guo H, Li H, Ling L, Gu Y, Ding W. Endoplasmic reticulum chaperon tauroursodeoxycholic acid attenuates aldosterone-infused renal injury. Mediators Inflamm. 2016;2016:4387031. doi:10.1155/2016/ 4387031

34. Lin JH, Li H, Zhang Y, Ron D, Walter P. Divergent effects of PERK and IRE1 signaling on cell viability. PLoS One. 2009;4(1):e4170. doi:10.1371/journal.pone. 0004170

35. Hetz C. The unfolded protein response: controlling cell fate decisions under ER stress and beyond. Nat Rev Mol Cell Biol. 2012;13 (2):89-102. doi: $10.1038 / \mathrm{nrm} 3270$

36. Lin JH, Li H, Yasumura D, et al. IRE1 signaling affects cell fate during the unfolded protein response. Science (New York, NY). 2007;318(5852):944-949. doi:10.1126/science.1146361

37. Giribabu N, Karim K, Kilari EK, Salleh N. Phyllanthus niruri leaves aqueous extract improves kidney functions, ameliorates kidney oxidative stress, inflammation, fibrosis and apoptosis and enhances kidney cell proliferation in adult male rats with diabetes mellitus. J Ethnopharmacol. 2017;205:123-137. doi:10.1016/j.jep.2017.05.002

\section{Publish your work in this journal}

Drug Design, Development and Therapy is an international, peerreviewed open-access journal that spans the spectrum of drug design and development through to clinical applications. Clinical outcomes, patient safety, and programs for the development and effective, safe, and sustained use of medicines are a feature of the journal, which has also been accepted for indexing on PubMed Central. The manuscript management system is completely online and includes a very quick and fair peer-review system, which is all easy to use. Visit http://www. dovepress.com/testimonials.php to read real quotes from published authors. 